\title{
Impact of corticosteroid therapy and surgery start time on blood glucose variability in patients undergoing
}

brain tumor surgery

Kazlauskas M., Banevicius G., Vilke A., Bilskiene D., Macas A.

Lithuanian University of Health Sciences, Dept. of Anaesthesiology, Kaunas, Lithuania

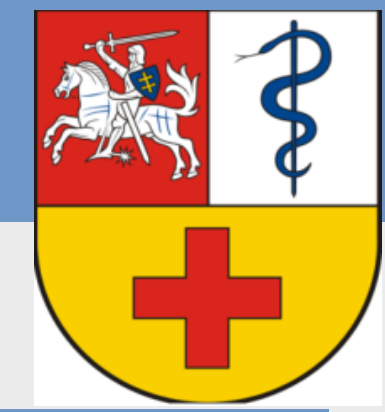

\section{BACKGROUND}

Most patients undergoing brain tumor surgery receive perioperative corticosteroids to reduce tumor-associated edema. However, it has been shown to cause hyperglycemia.

\section{GOAL OF THE STUDY}

The aim of our study was to evaluate influence of corticosteroids and surgery start time on blood glucose variability during operative day.

\section{MATERIALS AND METHODS}

The prospective observational study was carried out at a hospital of Lithuanian university of health sciences and 103 adults ASA I-III, aged 18-96, nondiabetics treated with dexamethasone before operation were involved. Patients underwent brain tumor surgery under general anaesthesia, maintained with sevoflurane. All patients received $8 \mathrm{mg}$ dexamethasone i.v. during anaesthesia induction. They were grouped by surgery start time: morning surgery (started to 12 p.m.) group I $(n=75)$, afternoon surgery

\section{RESULTS}

There were no significant differences between the groups regarding to demographic data (sex, age, BMI, ASA class), only the duration of anaesthesia was significantly longer in group I (Table 1).

Table 1. Demographic data and ASA class

\begin{tabular}{|c|c|c|c|}
\hline \multirow[b]{2}{*}{ Variable } & \multicolumn{2}{|c|}{ Surgery start time } & \multirow[b]{2}{*}{$P$ value } \\
\hline & $\begin{array}{c}\text { Group I } \\
(n=75)\end{array}$ & $\begin{array}{c}\text { Group II } \\
(\mathbf{n}=\mathbf{2 8})\end{array}$ & \\
\hline \multicolumn{3}{|l|}{ Sex: } & \multirow{3}{*}{ SI } \\
\hline Male & $34(45.3 \%)$ & $15(53.6 \%)$ & \\
\hline Female & $41(54.7 \%)$ & $13(46.4 \%)$ & \\
\hline Age (years) & $58.1 \pm 13.6$ & $62.0 \pm 12.8$ & SI \\
\hline BMI $\left(\mathrm{kg} / \mathrm{m}^{2}\right)$ & $26.1 \pm 5.1$ & $27.6 \pm 4.2$ & SI \\
\hline ASA class I & $1(1.3 \%)$ & - & \multirow{3}{*}{ SI } \\
\hline ASA class II & $42(56.0 \%)$ & $13(46.4 \%)$ & \\
\hline ASA class III & $32(42.7 \%)$ & $15(53.6 \%)$ & \\
\hline $\begin{array}{l}\text { Anaesthesia } \\
\text { duration (min) }\end{array}$ & $238.3 \pm 67.7$ & $205.2 \pm 38.5$ & $0.003^{*}$ \\
\hline $\begin{array}{l}\text { Operation } \\
\text { duration (min) }\end{array}$ & $165.3 \pm 54.7$ & $145.2 \pm 36.0$ & SI \\
\hline
\end{tabular}

Data are presented as mean \pm SD and $\mathrm{n}(\%)$.

*when $\mathrm{p}<0.05$ between groups

(started after 12 p.m.) group II $(n=28)$. Blood glucose concentrations were measured 5 times within 24 hours in operative day with calibrated glucose express meter: 09:00pm, day before operation, 07:00am, operation day, after anaesthesia induction, end of operation, 09:00pm, operation day. The data is presented as mean \pm SD. Statistical analysis was performed using Pearson's test, Friedman's test, Student's t test and $\chi^{2}$ test. $p<0.05$ was defined as significant.

Figure 1. Blood glucose levels

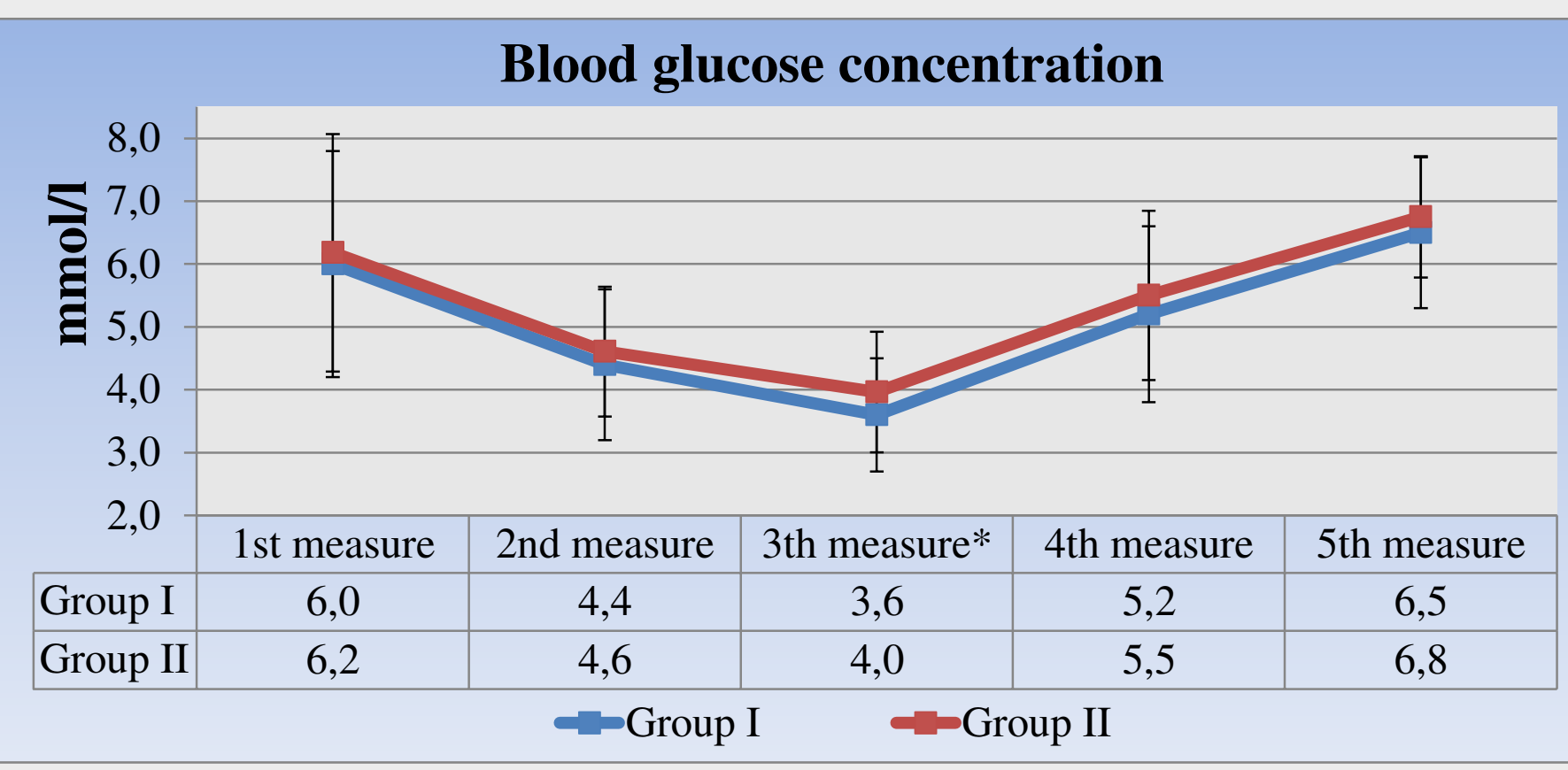

Data are presented as mean, error bar - standard deviation.

$*_{\text {when }} \mathrm{p}<0.05$ between groups.

Figure 2. Blood glucose changes in perioperative period

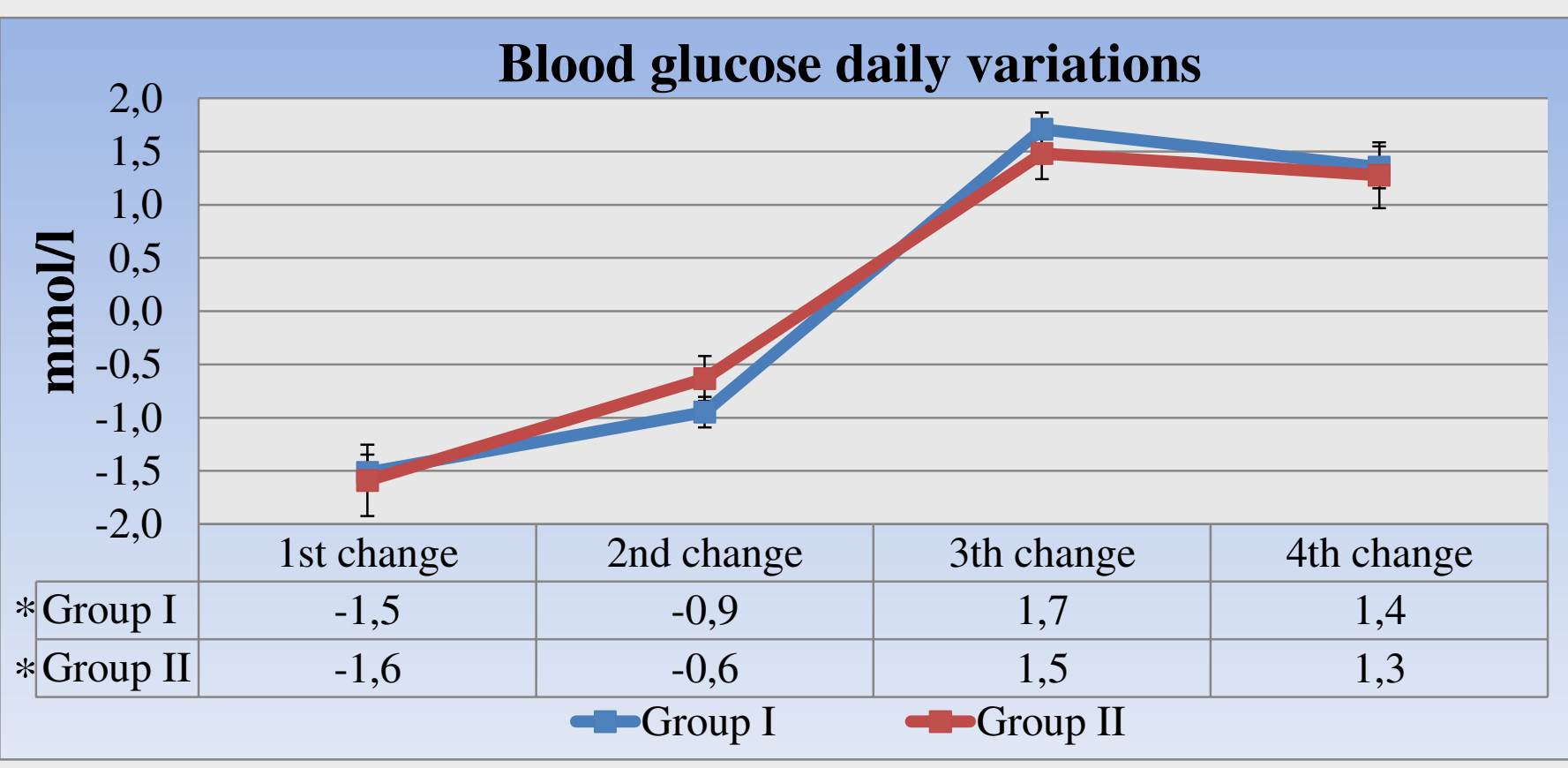

Data are presented as mean, error bar - standard deviation.

$* p<0.05$ in group at any measure.

group I ( $15 \pm 3.3 \mathrm{mg}$ vs. $13 \pm 3.0 \mathrm{mg}, \mathrm{p}<0.05)$.

Correlation between total dose of dexamethasone and blood dexamethasone did not differ significantly between groups (68.9 $\pm 5.0 \mathrm{mg}$ vs. $77.9 \pm 6.7 \mathrm{mg}, \mathrm{p}>0.05)$, but the total dose of dexamethasone on operation day was significantly higher in

\section{CONCLUSION}

The blood glucose level of patients with brain tumor and corticosteroid therapy has increased significantly during operation, but it has not depended on surgery start time. Identified blood glucose variations within did not require any correction.

\section{REFERENCES}

1. Dietrich J, Rao K, Pastorino S, Kesari S. Corticosteroids in brain cancer patients: benefits and pitfalls. Expert Rev Clin Pharmacol. 2011 Mar;4(2):233-42.

2. Godoy DA, Di Napoli M, Biestro A, Lenhardt R. Perioperative glucose control in neurosurgical patients. Anesthesiol Res Pract. 2012;2012:690362. 